\title{
Influence of Hospital Volume Effects and Minimum Caseload Requirements on Quality of Care in Pancreatic Surgery in Germany
}

\author{
Christian Krautz Axel Denz Georg F. Weber Robert Grützmann \\ Department of Surgery, University Medical Center Erlangen, Friedrich-Alexander-University Erlangen-Nürnberg, Erlangen, Germany
}

\section{Keywords}

Administrative data $\cdot$ Hospital volume .

Minimum caseload requirements · Pancreatic surgery .

Quality of care

\section{Summary}

Introduction: Numerous international studies have identified hospital volume as significant independent variable of death following pancreatic surgery. Most of these studies were limited to regions of countries or portions of a national population and did not include data on volume-outcome effects in Germany. Methods: The Medline database was systematically searched to identify studies that analyzed volume-outcome relationships and effects of minimum caseload requirements on outcomes of pancreatic surgery in Germany. Results: Recent observational studies utilizing German hospital discharge data confirmed that patients undergoing pancreatic surgery in Germany also have better outcomes when treated in facilities with high annual caseloads. Besides a decreased risk of in-hospital mortality, there is also a reduced risk of 1-year mortality in high-volume hospitals. In addition, there is evidence that adherence to already existing minimum caseload requirements reduces morbidity and mortality of pancreatic surgery in Germany. As a result of an insufficient centralization in the recent past, however, a large proportion of hospitals that perform pancreatic surgery still do not meet minimum caseload requirements. Conclusions: Specific measures (i.e. sanctions for failure to achieve minimum volumes) that initiate a sufficient centralization process without threatening patient access to surgical care are needed.

\section{Introduction}

A considerable amount of literature has been published on the volume-outcome relationship in pancreatic surgery. The majority of these studies have found a strong inverse association between mortality and hospital volume [1-5]. Moreover, a number of studies revealed similar effects between hospital volume and postoperative complications, readmission rates, and failure to rescue, respectively [6-8]. Despite this growing body of international evidence, equivalent outcomes data on pancreatic surgery in Germany have not been available until recently. Nonetheless, the German Federal Joint Committee (G-BA) has established minimum caseload requirements for pancreatic surgery (10 procedures per year) with the goal to improve patient care. This regulation has been formally binding for all hospitals that are permitted to charge the German statutory health insurance carriers for their services since 2004 [9]. However, the regulation also provides certain general exceptions that may compromise its desired effect of centralization of care structures. The fact that we unexpectedly found high mortality for most procedures and medical indications as well as high rates of postoperative complications in our recent analysis is additionally challenging the efficacy of current health policies that aim to improve the outcomes of high-risk surgical procedures in Germany [10].

Therefore, current research focuses on providing objective data on effects of volume-outcome relationships and minimum caseload requirements on the quality of surgical care in pancreatic surgery in Germany. This article reviews the most recent studies on this subject.

\section{Methods}

A literature search on outcomes of pancreatic surgery in Germany was carried out using the Medline database. The following search terms were used: ('pancreatic surgery' OR 'pancreatic resec-

\section{KARGER}

(C) 2017 S. Karger GmbH, Freiburg
Prof. Dr. med. Robert Grützmann, MBA 
tion' OR 'pancreatectomy') AND ('Germany') AND ('hospital volume' OR 'high volume' OR 'low volume' OR 'annual caseload') OR ('minimum caseload requirements' OR 'minimum volume requirements'). The search findings were screened and full articles were obtained in case of potentially relevant references. To be eligible for inclusion, studies had to report on volume-outcome relationships or effects of minimum volume requirements in pancreatic surgery in Germany. All studies were restricted to those analyzing datasets of mandatory hospital quality reports or hospital discharge data that cover the entire German population or large portions of it.

\section{Results}

A total of 57 studies were identified. After screening of titles and abstracts, 51 articles were excluded and of the remaining 6, 4 publications were suitable for inclusion in the present study [9, 11-13].

Using data from the mandatory hospital quality reports for the years 2006, 2008, and 2010, de Cruppé et al. [11] performed a retrospective longitudinal analysis to evaluate potential centralization effects for six procedures. For pancreatic surgery, no centralization was found from 2006 to 2010. Instead, the annual number of cases and the number of hospitals performing pancreatic surgery rose by 11.2 and $5.5 \%$, respectively. Moreover, about $30 \%$ of all hospitals that performed pancreatic surgery did not comply with minimum caseload requirements. An analysis of German hospital discharge data from 2006 to 2011 also revealed that a large proportion of hospitals perform pancreatic surgeries without achieving minimum caseload requirements and that a substantial number of pancreatic surgery inpatients was treated in such facilities [12]. This study found a mild trend towards a concentration of care structures within the field of pancreatic surgery from 2005 through 2011 (percentage of hospitals with caseload below minimum caseload requirements: 64.1 to $48.7 \%$; percentage of pancreatic surgery cases in hospitals below minimum caseload requirements: 19.4 to $11.4 \%$, respectively), which seemed to stop between 2011 and 2013 [9]. Another study assessed volume-outcome relationships related to pancreatic surgery using hospital discharge data from Germany's largest provider of statutory health insurance [13]. Although this provider covers only $30 \%$ of Germany's population, its database offers the possibility to follow up patients for up to 1 year beyond the initial inpatient episode. Based on this feature, Alsfasser et al. [13] were able to demonstrate that hospitals of the lowest volume quintile exhibited not only the highest in-hospital mortality (adjusted odds ratios (AOR): 2.1 (95\% confidence interval (CI): 1.4-3.0)) but also the highest 90-day mortality (AOR: 1.9 (95\% CI: 1.4-2.6)) and 1-year mortality (AOR: 1.7 (95\% CI 1.4-2.2)) compared to hospitals of the highest volume quintile. Finally, Nimptsch et al. [9] used nationwide German hospital discharge data to investigate the association between adherence to minimum caseload requirements and outcome of complex in-hospital procedures such as pancreatic surgery. In this study, all inpatient episodes of pancreatic surgery from 2006 to 2013 ( $n=78,879$ ) were assigned to two groups according to the annual caseload of the treating hospital $(<$ minimum caseload

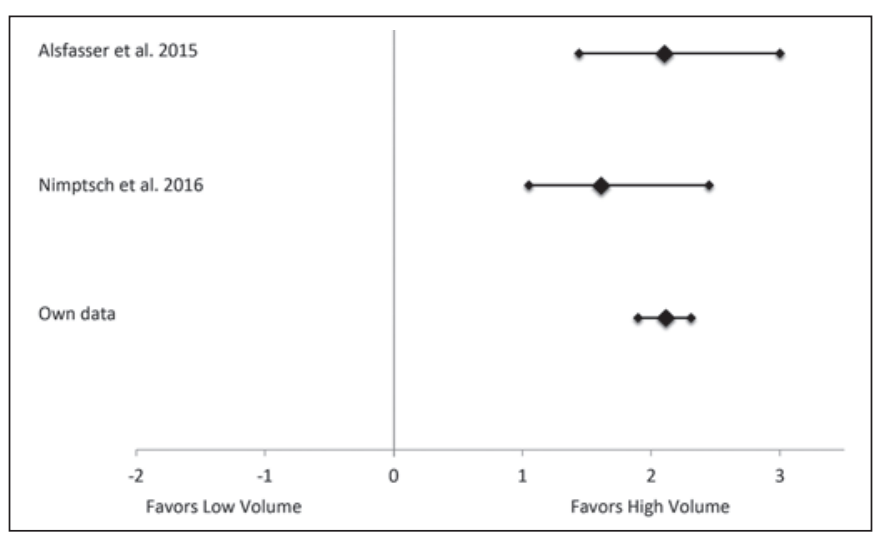

Fig. 1. Odds ratios for in-hospital mortality following pancreatic surgery in lower-volume compared to higher-volume hospitals.

vs. $\geq$ minimum caseload). Adjusted in-hospital mortality in hospitals with caseload above the minimum caseload requirements was significantly lower than in hospitals with a caseload below the minimum caseload (8.6\% (95\% CI: 8.3-8.8) vs. $11.8 \%$ (95\% CI: $11.2-$ $12.5)$ ). In addition to these studies, we confirmed the presence of a strong inverse association between hospital volume and in-hospital mortality (fig. 1) in an unpublished analysis of all German inpatient cases of major pancreatic surgery $(n=60,858)$ from 2009 to 2014 (i.e. risk-adjusted mortality of very-low-volume vs. very-high-volume hospitals: 11.5 (95\% CI: 10.9-12.1) vs. 6.5\% (95\% CI: 6.0-7.0)). Besides, we found a strikingly high number of hospitals (mean \pm standard deviation (SD): $398 \pm 8.5$ ) with a very low annual caseload (mean \pm SD: $5.1 \pm 3.7$ ) (fig. 2). In consequence, we simulated a potential market concentration within the lower (poorly performing) hospital volume quintiles and estimated that such a centralization process could potentially prevent 120 deaths per year based on the crude mortality rates (unpublished data).

\section{Discussion}

Recent observational studies provided two important findings. First, there is a strong inverse relationship between hospital volume and mortality following pancreatic surgery in Germany that persists for up to 1 year. Second, the introduction of minimum caseload requirements did not result in a sustainable consolidation of care structures in pancreatic surgery over the last years.

The existence of a significant relationship between hospital volume and mortality supports our previous assumption of the presence of a relevant publication bias wherein mostly departments with good performance report their results. While mortality rates reported by previous German single- or multi-institutional studies published on pancreatic surgery $(0-7.8 \%)$ correspond to higher hospital volume categories [14-18], nationwide in-hospital mortality $(10.1 \%)$ rather correlates with lower hospital volume categories [10]. These findings underline the importance of accompanying outcomes research that comprises data from large clinical registries or even complete national administrative databases, as they pro- 
Fig. 2. Annual distribution of hospitals that performed major pancreatic resections in Germany according to hospital volume quintiles (numbers within brackets represent the mean hospital volume \pm standard deviation of each hospital volume quintile) and annual overall in-hospital mortality from 2009 through 2014.

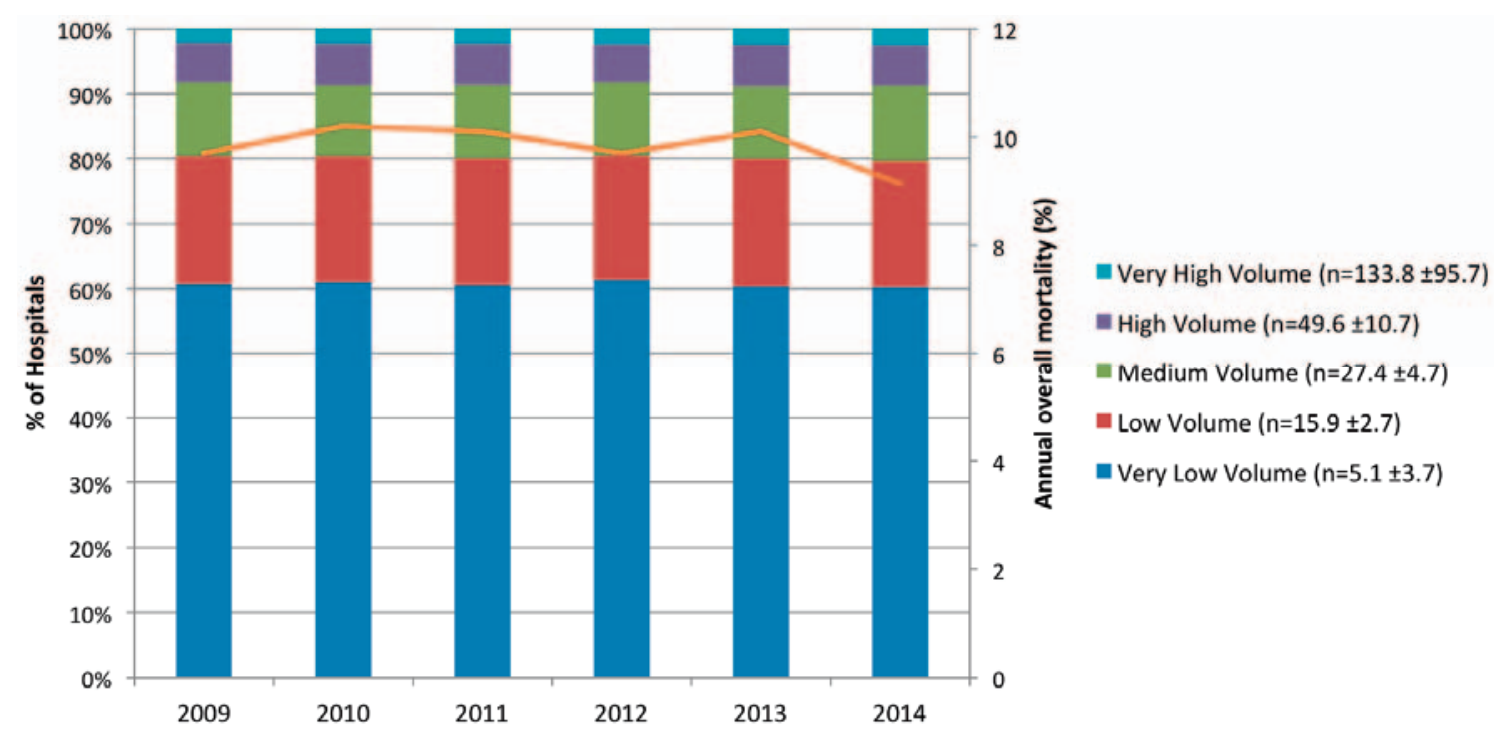

vide an objective view on disease-specific patterns of hospital care and on outcomes. Beyond that, volume-outcome effects are of special interest in complex surgery because quality parameters related to such interventions continue to remain a source of debate. For complex procedures that are less frequently performed, such as esophagectomy and pancreatectomy, direct measures of surgical outcomes such as mortality and morbidity rates will not be statistically reliable metrics for policy decisions [1]. The performance status ('good vs. poor quality') of hospitals with caseloads below 10 procedures per year (almost half of all hospitals performing pancreatic surgery in Germany) cannot be assessed due to the statistical limitations of small sample sizes (low caseloads). In these settings, hospital volume remains a useful proxy measure of surgical quality and allows for selective referral of patients to hospitals with aboveaverage performance.

The fact that almost half of all hospitals performing pancreatic surgery in Germany still miss minimum caseload requirements is not surprising, as Germany's inpatient care is characterized by overcapacities and too many small, non-specialized hospitals. Indeed, the hospital market structure did not keep up with the demands of increasing specialization and high-tech patient care resulting from recent medical advancements. Consequently, facilities with low caseloads may also fail to achieve equivalent outcomes like hospitals with high caseloads due to deficits in structural and process quality. A number of studies have demonstrated that variation in mortality is not just attributed to operative performance [6, 7]. Rates of postoperative complications alone also do not explain this discrepancy. Instead, variation in how well hospitals recognize and rescue patients from major complications once they have occurred is playing a more important role in explaining mortality rates [7]. The results of these studies show that outcomes following complex surgery also depend in large parts on the expertise of all persons and teams involved in complication management and not only on the surgeon's experience. This is particularly obvious concerning postpancreatectomy hemorrhage, the most feared compli- cation in pancreatic surgery, that is best treated by interventional radiology rather than surgery in most of the cases [19]. Therefore, a 24-h interventional radiology service is a must-have structural attribute for hospitals that perform pancreatic surgery.

In the past, studies on US databases have described an increasing regionalization of high-risk surgery including pancreatic resections as a result of volume-based referral initiatives [2,20]. There is evidence that increased market concentration and hospital volume have contributed to a declining mortality of pancreatectomy in the USA [2]. The studies mentioned above prove that referral patterns for pancreatic surgery have not sustainably changed since 2006 [9, 12]. Our present study confirmed that a centralization of care structures did not occur at the national level in Germany (fig. 2). Instead, a trend towards an increase of inpatient cases in all volume quintiles could be observed.

The fact that patients undergoing pancreatic surgery in verylow-volume hospitals and even low-volume hospitals still have a disproportionally high risk of mortality necessitates a rethinking in current health policies. From our point of view, the following measures would potentially reduce mortality: First, minimal caseload requirements have to be enforced effectively. This may include sanctions for failure to achieve minimum volumes. Second, exception regulations should be restricted to facilities with apparent structural changes and adequate preconditions. Third, the threshold value of minimum volume has to be increased as the current value (10 resections per year) is too low to effectively reduce the current national overall mortality rate $(10.1 \%)$, even if all hospitals comply with minimum volumes. Correspondingly, other European countries have already implemented higher minimum volumes (i.e. Netherlands $=20$ resections per year, France $=30$ resections per year).

Despite justified efforts to consolidate care structures of pancreatic surgery in Germany, one should keep in mind that volumebased referral may also affect the total number of procedures performed. Emphasis on procedure volume as a quality indicator may create incentives for hospitals and surgeons to operate more often 
[21]. Moreover, indirect effects of volume-based referral could interfere with patient access to surgical care, since loss of caseloads may threaten the viability of hospitals and negatively affect their ability to recruit and retain surgeons. Therefore, policy makers should anticipate potential benefits as well as unintended side effects of volume-based referral strategies.

\section{Conclusion}

Recent studies utilizing large administrative databases verified that pancreatic surgery in Germany is also subject to strong, inverse volume-outcome relationships (i.e. patients treated in highervolume facilities have better outcomes). There is objective evidence that compliance with existing minimum caseload requirements would already reduce morbidity and mortality of pancreatic surgery in Germany. As a result of an insufficient centralization in the recent past, however, a large proportion of hospitals that perform pancreatic surgery still do not meet minimum caseload require- ments. Therefore, specific measures as well as new strategies to initiate sufficient centralization in the field of pancreatic surgery without threatening patient access to surgical care are needed. This process should be accompanied by initiatives to improve structural or organizational factors (proficiency in treatment of critical ill patients, minimal standards for nurse staffing) as well as specific processes of care (implementation of guidelines to manage procedurespecific complications).

\section{Acknowledgement}

CK performed the literature search and wrote the manuscript. AD, GW, and RG edited the manuscript and provided intellectual input.

\section{Disclosure Statement}

$\mathrm{CK}, \mathrm{AD}, \mathrm{GW}$, and RG have no conflicts of interest to disclose.

\section{References}

1 Reames BN, Ghaferi AA, Birkmeyer JD, Dimick JB Hospital volume and operative mortality in the modern era. Ann Surg 2014;260:244-251.

2 Finks JF, Osborne NH, Birkmeyer JD: Trends in hospital volume and operative mortality for high-risk surgery. N Engl J Med 2011;364:2128-2137.

3 Lemmens VE, Bosscha K, van der Schelling G, Brenninkmeijer S, Coebergh JW, de Hingh IH: Improving outcome for patients with pancreatic cancer through centralization. Br J Surg 2011;98:1455-1462.

4 Gani F, Azoulay D, Pawlik TM: Evaluating trends in the volume-outcomes relationship following liver surgery: does regionalization benefit all patients the same? J Gastrointest Surg 2017;21:463-471.

5 van Heek NT, Kuhlmann KFD, Scholten RJ, et al: Hospital volume and mortality after pancreatic resection. Ann Surg 2005;242:781-790.

6 Amini N, Spolverato G, Kim Y, Pawlik TM: Trends in hospital volume and failure to rescue for pancreatic surgery. J Gastrointest Surg 2015;19:1581-1592.

7 Ghaferi AA, Birkmeyer JD, Dimick JB: Variation in hospital mortality associated with inpatient surgery. N Engl J Med 2009;361:1368-1375.

8 Brooke BS, Goodney PP, Kraiss LW, Gottlieb DJ, Samore MH, Finlayson SR: Readmission destination and risk of mortality after major surgery: an observational cohort study. Lancet 2015;386:884-895.
9 Nimptsch U, Peschke D, Mansky T: Minimum caseload requirements and in-hospital mortality: observational study using nationwide hospital discharge data from 2006 to 2013 (Article in German). Gesundheitswesen 2016;DOI: 10.1055/s-0042-100731.

10 Nimptsch U, Krautz C, Weber GF, Mansky T, Grützmann R: Nationwide in-hospital mortality following pancreatic surgery in Germany is higher than anticipated. Ann Surg 2016;264:1082-1090.

11 de Cruppé W, Malik M, Geraedts M: Minimum volume standards in German hospitals: do they get along with procedure centralization? A retrospective longitudinal data analysis. BMC Health Serv Res 2015;15:279.

12 Peschke D, Nimptsch U, Mansky T: Achieving minimum caseload requirements - an analysis of hospital discharge data from 2005-2011. Dtsch Arztebl Int 2014;111:556-563.

13 Alsfasser G, Leicht H, Günster C, Rau BM, Schillinger G, Klar E: Volume-outcome relationship in pancreatic surgery. Br J Surg 2015;103:136-143.

14 Distler M, Rückert F, Hunger M, et al: Evaluation of survival in patients after pancreatic head resection for ductal adenocarcinoma. BMC Surg 2013;13:12.

15 Hartwig W, Gluth A, Hinz U, et al: Total pancreatectomy for primary pancreatic neoplasms: renaissance of an unpopular operation. Ann Surg 2015;261:537-546.
16 Wellner UF, Kulemann B, Lapshyn H, et al: Postpancreatectomy hemorrhage - incidence, treatment, and risk factors in over 1,000 pancreatic resections. J Gastrointest Surg 2014;18:464-475.

17 Uzunoglu FG, Reeh M, Vettorazzi E, et al: Preoperative Pancreatic Resection (PREPARE) score. Ann Surg 2014; 260:857-864.

18 Keck T, Wellner UF, Bahra M, et al: Pancreatogastrostomy versus pancreatojejunostomy for RECOnstruction after PANCreatoduodenectomy (RECOPANC, DRKS 00000767). Ann Surg 2016;263:440-449.

19 Grützmann R, Rückert F, Hippe-Davies N, Distler M, Saeger H-D: Evaluation of the International Study Group of Pancreatic Surgery definition of post-pancreatectomy hemorrhage in a high-volume center. Surgery 2012;151:612-620.

20 Gasper WJ, Glidden DV, Jin C, Way LW, Patti MG: Has recognition of the relationship between mortality rates and hospital volume for major cancer surgery in California made a difference?: A follow-up analysis of another decade. Ann Surg 2009;250:472-483.

21 Goodney PP, Stukel TA, Lucas FL, Finlayson EVA, Birkmeyer JD: Hospital volume, length of stay, and readmission rates in high-risk surgery. Ann Surg 2003; 238:161-167. 